Bolm Inst. oceanogr., S Paulo, 28(2):55-64,

\title{
TRES DINOFLAGELADOS NUEVOS O INTERESANTES DE AGUAS BRASILEÑAS
}

\author{
ENRIQUE BALECH*
}

Museo Argentino de Ciencias Naturales "Bernardino Rivadavia"

\section{Synops is}

The author describes two new species: Protoperidinium steidingerae and Gonyaulax kutnerae, and a third, little known species, Protoperidinium norpacense Balech. The three of them were collected in plankton tows in the South Brazilian littoral. The present study of $P$. norpacense completes the original description and shows some differences with the thecae from the tropical Pacific. Its relationships with $P$. heteracanthum Dangeard are discussed. P. steidingerae has been described by Steidinger \& Williams as P. oblongum. It is close to $P$. venustum but it has several very peculiar features: pointed anterior horn; first apical plate conspicuously shorter than the epitheca; neither 1' nor $3^{\prime}$ reach the apex where $2^{\prime}$ is largely connected with $4^{\prime}$ on the ventral side; seemingly there are no platelets $P$ and $X$. Gonyaulax kutnerae belongs to the tamarens is group: its size, shape and weak thecawalls and tabular details tell it apart from the other species of the group. The only species which is somewhat alike is G. brevisulcatum Dangeard which is almost a nomem nudum. Recently Taylor (1976) described as G. brevisulcatum a species which clearly differs from $G$. kutnerae and which seems to be a Peridiniella.

\section{Introducciōn}

La Dra. M. B. Kutner, de1 Instituto Oceanogräfico da Universidade de São Paulo, me permitió revisar cierto nümero de muestras recogidas por el buque oceanogräfico "Prof.W. Besnard" durante dos cruceros en el año 1976 (FINEP III, en mayo, FINEP IV, en setiembre-octubre) y otro en enero de 1977. E1 área investigada se extiende en el litoral brasileño desde aproximadamente Cabo Frio hasta la is la de Santa Catarina, es decir desde aproximadamente $23^{\circ} \mathrm{S}$ a $28^{\circ} \mathrm{S}$.

Entre otro material interesante he podido distinguir dos especies, una de Protoperidinium y otra de Gonyaulax, que resultan nuevas para la ciencia aunque el Protoperidinium ya fue observado antes pero no bien identificado. Una tercera especie del último género no es inédita pero si interesante y poco conocida; sus ejemplares presentan caracteres un poco distintos de los de su primera publicación.

\section{Material y mëtodos}

Los especímenes estudiados fueron aislados con micropipetas, observados, dibujados y medidos en distintas posiciones y luego cada teca fue desarticulada con

* Miembro de la carrera del investigador del Consejo Nacional de Investigaciones Cientîficas y Técnicas, Argentina.

Publ. nọ 452 do Inst. oceanogr. da Usp. ayuda del hipoclorito de sodio para el estudio tabular, que se hizo bajo objetivo de inmersión homogénea, Los símbolos y nomenclatura usados son los de uso corriente, salvo para Gonyaulax en que la placa hipotecal generalmente considerada intercalar o placa $P$ es 11 amada primera antapical (1'''') por las razones expuestas por el autor en la Conferencia Penrose sobre dinoflagelados (1978). Las medidas son en micrones.

Descripciōn de las especies

Protoperidinium steidingerae n.sp. (Läm. 1, Figs 1-6; Läm. 2, Figs 1-10) Peridinium oblongum, Steidinger y Williams, 1970: 57, fig. $107 \mathrm{a}, d$; non Peridinium oblongum (Aurivillus) Cleve. Especie grande, ortho hexa, planozona descendente (aproximadamente 1), del tipo de $P$. oceanicum, con cuernos bien desarrollados. Aplastamiento dorso-ventral fuerte. Cingulum bien inclinado respecto a1 eje longitudina1; āngulo äntero-ventral, formado por el cingulum y el eje, de unos $110^{\circ}$ (108-118). Cuernos muy desarrollados aunque bastante variables. Los antapicales son largos, de base gruesa, pontiagudos y divergentes; sus bordes internos forman una concavidad bastante amplia y generalmente asimétrica; con frecuencia el borde interno del cuerno derecho es más cóncavo que el del izquierdo y también muchas veces el derecho es 
más grueso que el otro. Lo más característico es, sin embargo, el apical: aunque muy variable, en la mayoría de los individuos es muy largo y en todos termina en punta.

La placa 1', rómbica, tiene los dos triāngulos subiguales aunque con frecuencia se nota que el inferior es algo más corto: el borde póstero-derecho siempre es muy sinuoso y los restantes suelen ser casi rectos. Pero 10 notable de esta placa es su relativa cortedad que hace que su extremo anterior quede 1 ejos del äpice, completamente desconectado de éste. E1 ápice puede ser completamente puntiagudo o mostrar dos puntas diminutas correspondientes a las placas $2^{\prime}$ y $4^{\prime}$. Estas dos apicales se unen ventralmente por un borde bastante largo y que, cerca de la extremidad del cuerno, se

Lämina 1 - Protoperidinium steidingerae. Figs $1,3,4$ y 6 , cuatro individuos en vista ventral; Fig. 2, placas epitecales dorsales; Fig. 5, vista dorsal del cuerno apical.

Todas las figuras a aproximadamente $\times 700$.

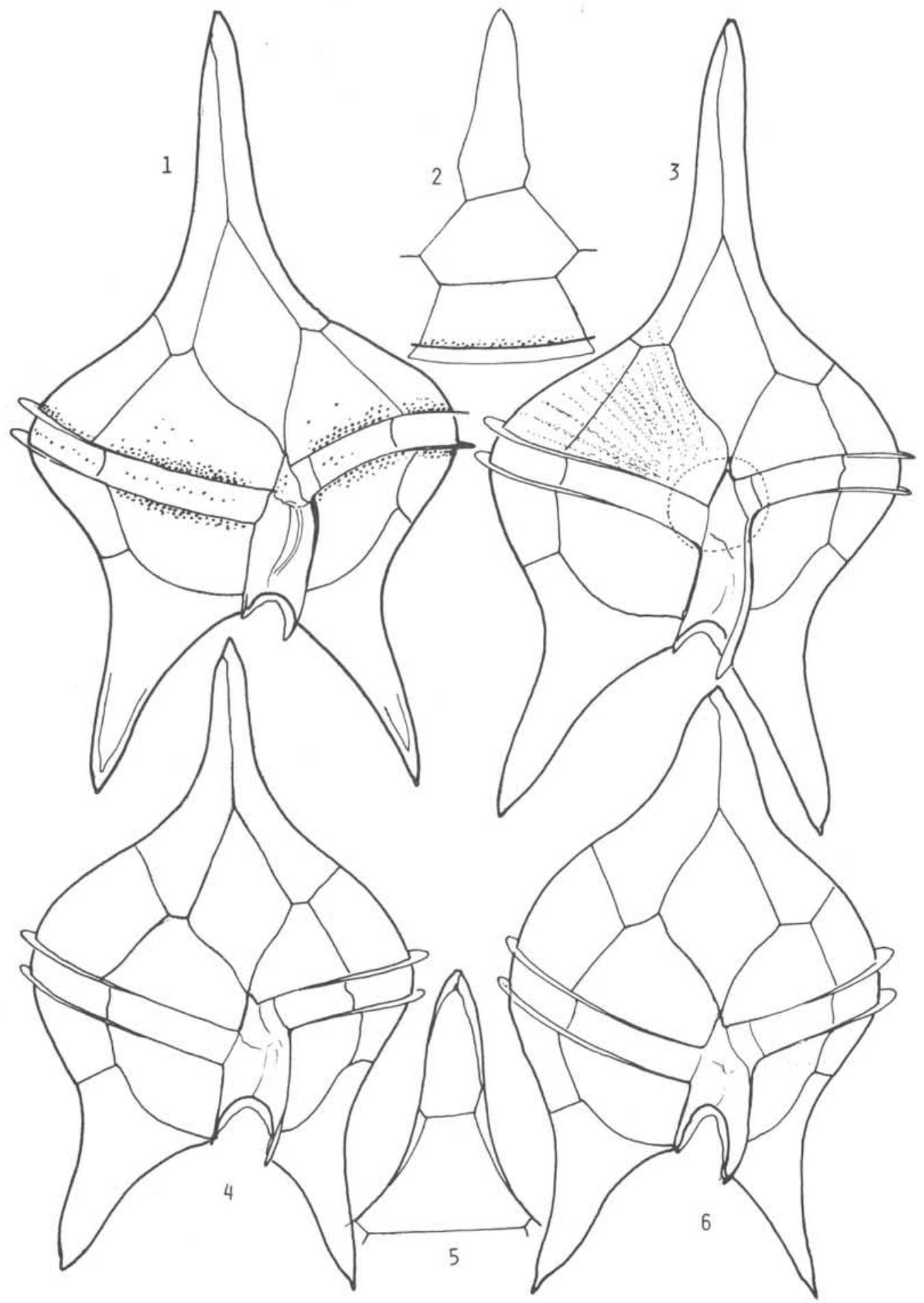


desvía hacia la derecha de modo que la cara ventral del ápice está constituída sólo por 1a $2^{\prime}$. No he podido detectar una placa $X$ y tampoco, con seguridad, una Po. Siguiendo el proceso de desarticulación de la teca con objetivo de inmersión pude ver, en un caso, un bastoncito diminuto que puede representar tal placa. Añado que, por 10 menos en algunas tecas, la linea de unión entre $2^{\prime}$ y $4^{\prime}$ se desvía con bastante brusquedad hacia 1a derecha y pasa hasta casi la cara dorsal para terminar en una pequeña abertura por debajo del ápice.

En verdad tampoco la $3^{\prime}$ llega hasta e1 mismo ápice que aparece entonces constituído por tan sólo dos placas: las dos laterales que en esta especie son también ventrales y dorsales.

La placa $2^{\text {a }}$ es hexagonal asimétrica; 1a longitud de sus bordes póstero-laterales es aproximadamente la mitad de la que tienen los ántero-laterales; borde anterior con frecuencia inclinado.

La 1''' es bastante angosta, alta y tiene el borde que la conecta con $1^{\prime \prime \prime}$ muy curvo, convexo; el interno, reforzado y cóncavo, sostiene una aleta sulcal más bien angosta, de ancho uniforme aunque muestra, cuando se 1a aplasta, una fuerte ondulación a media altura.

La $5^{\prime \prime \prime}$ es mucho más ancha y algo mảs baja; su borde posterior es también curvo, aunque de convexidad algo menos acentuada en algunas tecas; borde interno reforzado y un poco ondulado. 3''' a1ta, algo asimétrica.

Las dos antapicales forman los cuernos ya descritos los que a veces proyectan una espinita terminal.

Cingulum bordeado por membranas 1 is as de desarrollo mediocre. Placas extremas de ancho igual o casi igual al de las respectivas pre y postcingulares.

E1 sulcus, bien excavado, es de ancho casi uniforme; penetra muy poco en la epiteca. Su aleta terminal posterior-izquierda está formada por dos yuxtapuestas: 1a de 1a placa 1'''' y la de la S.p.

Placa T más bien ancha, algo variable, formada por una porción anterior más grande, cuadrangular de bordes algo curvos, y otra posterior triangular, bastante más corta y delgada, que resulta de la proyección del ángulo póstero-interno.

S.a. corta. Mitad anterior triangular con extremo anterior bastante puntiagudo; apófisis posterior grande y compleja.

La forma de la S.d. se ve en las Fi- guras. Tiene rudimento de cuello, a veces no destacado de la apófisis anterior. Borde interno más o menos convexo en 1a mitad anterior, cóncavo en la posterior donde sostiene una aleta curvada, angosta, más bien como un espesamiento; de su parte posterior se proyecta por 1a cara dorsal una formación triangular que se dirige hacia la derecha.

S.s. en $L$ bastante corta, con rama posterior muy desarrollada y cuyo ancho es generalmente igual a la altura de la placa. Borde interno regularmente cóncavo, con refuerzo bastante fuerte.

S.p. de tipo III, con rama izquierda mucho más ancha que 1a derecha,terminada en punta hacia adelante y con fuerte convexidad posterior; rama media o cuerpo muy delgado.

La S.m. es ancha y bastante fuerte. Junto a e1la hay, como en otras especies de1 "grupo oceanicum", una placa de refuerzo o S.d.i. La S.p.a. es grande.

Las placas generales tienen un reticulado tenue, de malla bastante pequeña y poros más visibles. Los poros forman siempre campos muy densos junto al borde cingular, tanto en las placas pre como postcingulares. En algunos casos son en las primeras tan densos que los poros quedan separados unos de otros por espacios del mismo ancho o aun menores que e1 diámetro de los poros.

E1 protoplasma es claro y suele tener cordones que irradian del centro, muy finos y poco aparentes. E1 núcleo es esferoidal o elíptico ancho, poco notable (hubo que teñirlo para ubicarlo con claridad) y situado a nivel de 1a región crucial. A nivel del borde de unión de 2' y 4' siempre se ve una acumulación de gränulos baciliformes.

Dimensiones: L 117,5-182, la mayoría entre 130 y 160 ; longitud aproximada de1 apical 26-60; Trd. 64-88; separación de1 extremo de los antapicales 46-67.

\section{Variaciones y discusiōn}

Las variaciones son importantes y se refieren tanto a las dimensiones absolutas como a 1 as relativas. Son sobre todo muy aparentes 1as de 1a longitud de los cuernos, en especial del apical y el grosor de los mismos; en pocos casos el apical (Lám. 2, Fig.3) es muy delgado y de diámetro casi uniforme en la mayor parte de su extensión. Aunque generalmente es largo se encuentran tecas en las que es bastante corto y hasta muy corto y robusto (Lám. 1, Fig. 6). La diver- 
gencia de los antapicales es también bastante variable y el contorno de la teca es más o menos ensanchado a nive1 del cingulum donde siempre es, en todo caso, bastante redondeado.

De las placas sulcales las más variables parecen ser las dos grandes laterales S.d. y, sobre todo, la S.s. Tambiēn varía bastante 1 a $T$; la S.a., en cambio, parece tener forma muy constante.

Esta especie fue en realidad primero figurada por Steidinger \& Williams (1970); en sus figuras se 1 a reconoce muy bien. Esos autores la presentaron bajo el nombre de $P$. oblongum. Más tarde Steidinger (in litt.) insistió en la independencia de $P$. oblongum respecto a $P$. oceanicum. No cabe duda de que su $P$. oblongumes una especie independiente, pero no corresponde al oblongum de los demás autores, por 10 menos de los que 1o dibujaron. En realidad $P$. oblongum es casi un nomen nudum ya que su creador no dio figuras ni una descripción útil. Esto dio pie a distintas interpretaciones de oblongum, pero todos 1o demás autores, aun los que mantienem su independencia, dieron este nombre a tecas que no se diferencian de $P$. oceanicum por ningún carácter fundamental, salvo su tamaño menor, forma menos gräcil y cuernos más cortos. En cambio la especie presentada por Steidinger \& Williams es inconfundible y con una serie de caracteres distintivos muy claros que hacen que se la individualice sin dificultad a pesar de las variaciones que experimenta.

La especie más próxima a $P$. steidingerae es $P$. venustum Matzenauer. Se $1 \mathrm{e}$ asemeja algo por la forma, el tamaño de los cuernos antapicales y 1 a concavidad interna de estos y, en parte, por el tipo de placas sulcales que, sin embargo, tienen detalles que separan con nitidez ambas especies. $P$. venustum es quadra, de forma más cuadrilátera, más angulosa a nivel del cingulum, tiene apical de tipo normal y la S.a., más larga, penetra profundamente en 1a epiteca; además tiene el protoplasma con cordones más gruesos y visibles.

Es en verdad una especie de muy alto interés por una serie de detalles tabulares muy peculiares: su apical puntiagudo, su falta (aparente?) de placas $P_{0}$ $\mathrm{y} X \mathrm{y}$, sobre todo, por $1 \mathrm{a}$ muy visible desconexión de $1^{\prime}$ con el ápice y la 1 arga unión ventral de $2^{\prime}$ y $4^{\prime}$.

Tengo el placer de dedicar la especie a uno de los codescubridores, la Dra. K. A. Steidinger, estudiosa de dinoflagelados.
Parece ser especie frecuente en el litoral del sur de Brasil. Se halla en muchas de las muestras comunicadas por la Dra. Kutner. Probablemente especie nerítica común el Atlāntico sudoccidental tropical y en el Golfo de México. Seguramente será identificada en otros mares cálidos.

En 1a estación 24 del primero de 10 s cruceros, y en las $75,79,82,83,96$ y 97 del último. Como no se hizo un estudio cuidadoso de todas 1 as muestras posiblemente se la encuentre mäs adelante en otras.

\section{Protoperidinium norpacense Balech}

$$
\text { (Läm. 2, Figs 11-20) }
$$

Peridinium norpacense Balech, 1962: 149, Läm. 20 (302-306).

Protoperidinium norpacense (Balech)

Balech, 1974:67.

Mediana a pequeña, globosa a elíptica ancha irregular, sin cuernos antapicales, con cuello muy pequeño, cilíndrico o algo cónico. Para hexa planozona ascendente (aproximadamente 1).

La hipoteca puede ser redondeada atrás o tener allí un aplastamiento y aun una ligera concavidad en la región antapical. Sulcus muy poco excavado, bastante corto, bordeado por aletas izquierda y derecha. Dos espinas antapicales muy desiguales, de implantación bastante ventral y oblicuas, por lo que, con la teca bien orientada para una vista ventral normal sólo se proyecta por detrás del cuerpo parte de la izquierda. Esta espina es fina pero larga y fuerte y está bordeada por aletas angostas. La derecha es corta.

Las placas $1^{\prime \prime}$ y $7^{\prime \prime}$ son bajas. E1 borde interno de 1 a $7^{\prime \prime}$ ' es convexo, muy oblicuo y 1lega a ser casi horizontal en su mayor extensión. $3^{\prime}$ bastante pequeña. Cuello sólo formado por la Po, cuya base además se expande bastante.

Postcingulares 1''' y 5 '' trapécicas; la primera bastante angosta. Ambas $11 \mathrm{e}-$ van aletas sulcales; 1 a de 1 ''', de borde libre irregular, se expande hacia atrás, donde 1lega a ser bastante ancha; 1a de $5^{\prime \prime \prime}$ es, en cambio, muy angosta. $3^{\prime \prime}$ casi simétrica.

Las dos placas antapicales tienen el borde interno bordeado por una especie de membrana o proyección de la capa externa de la teca que sostiene las espinas que, por 1o tanto, aparecen como no implantadas directamente en la pared tecal principal. La espina izquierda tiene como una espinita accesoria en la base y está bordeada por una membrana o aleta verda- 
Lämina 2 - Figs 1-10, Protoperidinium steidingerae. Fig. 1, vista lateral derecha; Fig. 2, placa 5''l (arriba) y l'''; Fig. 3, vista ventral de una epiteca de apical largo y de diămetro casi uniforme; Fig. 4, S.a.; Fig. 5, placas T; Fig. 6, tres S.s.; Fig. 7, una S.d.; Fig. 8, S.d., S.d.i., S.m. y S.p.; Fig. 9, una S.p. aplastada; Fig. 10, S.p.a.

Figs 11-20, Protoperidinium norpacense. Fig. 11 y 13, dos ejemplares en vista ventral (el 11 algo deformado por el desarrollo de las bandas articulares); Fig. 12, placas epitecales dorsales; Fig. 14, extremo interno de 5'1' (arriba) y 1111 ; Fig. 15, borde interno de 211'; Fig. 16, Po en yista apical y lateral; Fig. 17, l' '1 mostrando la implantación de la espina en un desdoblamiento membranoso; Fig. 18, cara interior del cuerpo de la S.d.; Fig. 19, teca en vista lateral izquierda; Fig. 20, sulcales y $T$.

Figuras $1,3,11-14$ y 19 a aproximadamente $\times 700$; las demäs a aumentos no calculados.

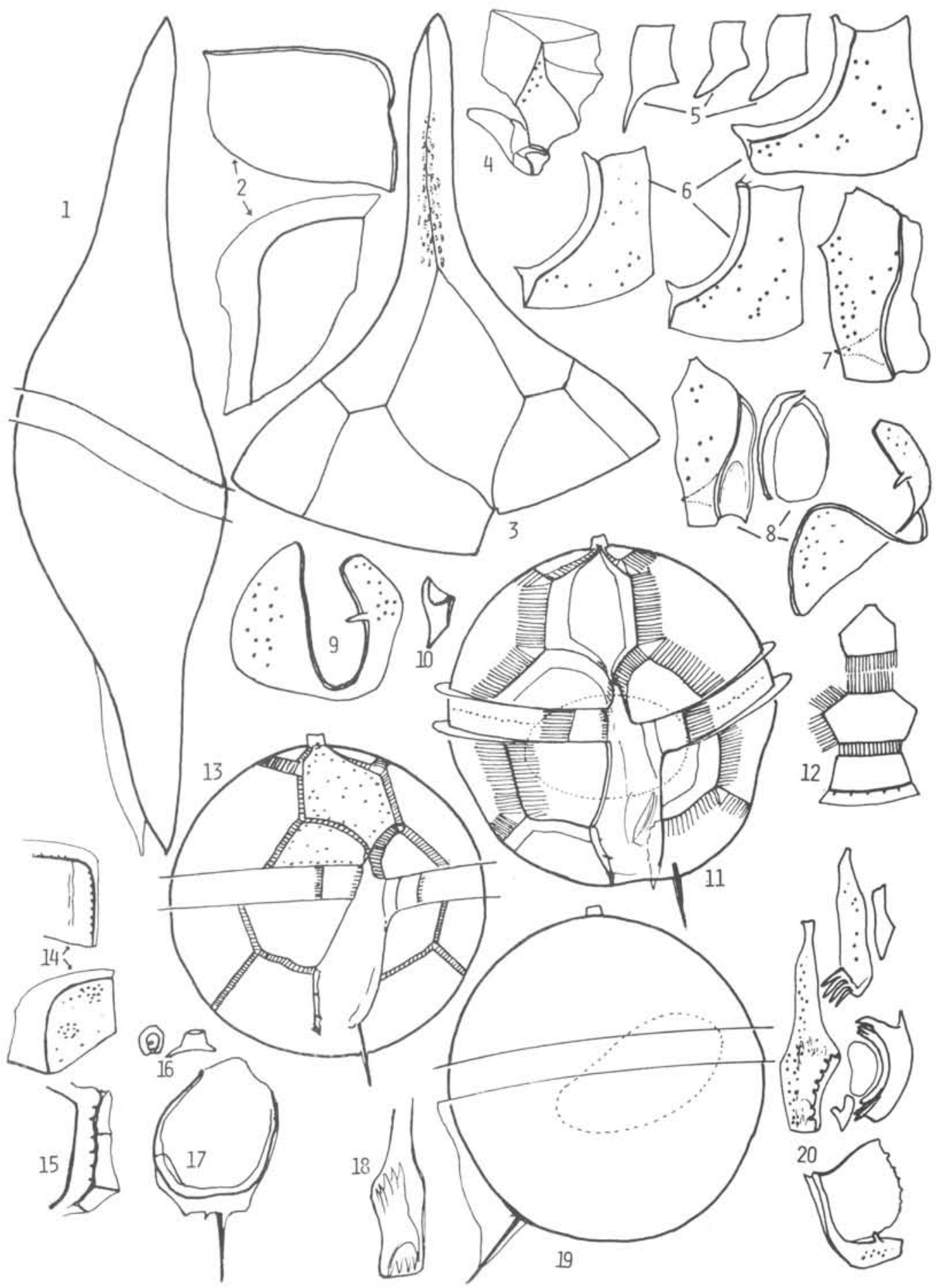


Bolm Inst. oceanogr., S Paulo, 28(2), 1979

dera angosta. En la 2'''' ese desdoblamiento tecal tiene un borde libre un poco cóncavo irregular, algo reforzado, con dentículos basales y sostiene una aleta con tres refuerzos espiniformes, uno anterior y dos posteriores de los que el de más atrás es muy corto. En la teca íntegra y en ciertas posiciones la observación atenta descubre estas dos espinas posteriores, una de ellas un poco más ventral y anterior que la otra.

Junto a la espina izquierda se ve la proyección de 1a amplia membrana de 1 a S.p., de borde denticulado.

Cingulum bordeado por membranas bien desarrolladas (ancho de unos $6 \mu$ ), casi lisas, pues solo tienen refuerzos basales cortos, finos y bastante espaciados. En algunos casos el borde libre de las aletas tiene pseudorradios débiles y cortos. Placas extremas bastantes angostas. En 1a $\mathrm{C}_{2}$ se nota una franja media de poros densos.

La teca es de paredes gruesas, de estructura claramente laminar. Suturas siempre en bandas estriadas más o menos amplias. La única escultura son poros de distribución irregular, bastante fuertes, que forman campos más o menos densos sobre todo cerca de los bordes de las placas. En la $1^{\prime \prime}$ con frecuencia se ven dos grupos de poros densos bastante bien definidos: uno cerca del borde anterior y otro a media altura, más cerca del lado interno que del externo.

Placa $T$ angosta, en forma de alfanje. S.a. más bien angosta, de longitud mediana con rama posterior que primero se dirige oblicua hacia la derechay adelante y luego se dobla bruscamente hacia atrás; esta parte distal está dividida en largos dientes, 4 o 5, que le dan aspecto de rastrillo.

S.d. larga, angosta, con cuello bien marcado y, en casi todas las tecas, un poco dilatado adelante. Apófisis anterior más 1 arga que el cuerpo propiamente dicho. Borde interno de éste con un refuerzo cóncavo que emite denticulaciones hacia el interior de la placa, bien visibles. En la cara interior o dorsal de 1a placa hay un espesamiento o membrana que ocupa casi todo el ancho del cuerpo y tiene los extremos divididos en forma de dientes largos.

S.s. muy angosta. E1 refuerzo interno se desdobla en ambos extremos en varias ramas bastante largas. Angulo ántero-externo bastante proyectado pero no filiforme.

S.p. de tipo IV. Rama derecha baja y corta. La izquierda, en cambio, es 1arga y sostiene una aleta amplia de borde libre denticulado.

E1 nücleo es esferoidal o elipsoidal ancho y se sitúa a nivel del cingulum o un poco por detrás.

Dimensiones: L 55-62; L.t. 61-68,5; cue11o 2-2,5; Trd.53-58; DV casi siempre igual al Trd. La longitud real de la espina izquierda es de unos 11-12. En algunos casos 1a longitud del cuerpo sin e1 cuello es igual o casi igual al transdiámetro, pero generalmente lo supera. Cuando la teca es netamente alargada suele serbastante irregulary los flancos de 1a hipoteca se muestran un poco aplastados en la media; esas tecas largas son las que tienen bandas más anchas.

\section{Discusiön y comparaciones}

Desde su descubrimiento no fue más citada, pero una descripciōn anterior de Matzenauer (1933: 480, fig. 73) 1a presentó como $P$. heteracanthum Dangeard. No hay dudas de que $P$. heteracanthum y P. norpacense estān muy emparentadas, pero la primera es de cingulum nivelado con aletas angostas; su sulcus es recto y muy angosta, hay menor desproporción entre las espinas y la izquierda es más corta, ampliamente alada y casi horizontal. Las dos placas precingulares ventrales son significativamente más disímiles entre si. E1 borde lateral derecho de $1^{\prime}$ es bastante más corto que en norpacense.

P. heteracanthum sec. Taylor (1976: 164, fig. 352,353 y 357 ) no es 1 a especie de Dangeard (1927a). Podría ser $P$. norpacense pero 11ama $1 \mathrm{a}$ atención en sus dibujos el enorme desarrollo de 1 a membrana de la S.P. que, aparentemente se extiende mucho más atrás que la espina izquierda que, además, sería muy corta y de longitud menor que la derecha.

P. norpacense fue primero descrita sobre material obtenido en una estación del Pacífico tropical. Estaba constituído por tecas perfectamente esféricas y en promedio algo más pequenãs que éstas. Casi seguro las diferencias se deben, sobre todo, al desarrollo de bandas suturales más o menos amplias del material brasileño, pues los ejemplares más pequeños y esféricos de êste son los que tienen bandas estrechas; los más grandes, más o menos irregularmente elipsoidales anchos, tienen bandas muy amplias .

E1 nuevo material amplía bastante 
1a descripción primera y da detalles de las placas sulcales que, por imperfección del estudio, no fueron figuradas en mi trabajo de 1962.

Abundante en la muestra 97 del ültimo crucero. Con este hallazgo hasta ahora $P$. norpacense se registró en aguas cálidas del Pacífico, del Indico y del Atlāntico Sudoccidental.

Gonyaulax kutnerae $n$. sp.

(Lām. 3, Figs 1-11)

Una de las especies mayores del "grupo tamarensis". Forma globosa con la epiteca algo cónica sin cuello ni hombros. Muy 1igero aplastamiento dorsoventral. Hipoteca hemisfërica un poco irregular sin concavidad antapical, espinas ni aletas proyectadas atrás. Cingulum bien excavado, descendente $(1-1,5)$. Sulcus amplio y relativamente corto. Fórmula tabular Po, 4', $6^{\prime} ', 6 \mathrm{C}, 6^{\prime}{ }^{\prime}, 2^{\prime \prime \prime}$ (o $1^{\prime \prime \prime}$ ' y $P$ en $1 \mathrm{a}$ nomenclatura utilizada hasta ahora), $8 \mathrm{~S}$.

Placa Po grande, oval algo deformada a la derecha, con e1 polo menor dirigido a 1a izquierda y ventralmente. Refuerzo interno, a corta distancia del margen; entre ambos una hilera de granulaciones fuertes (o una serie de depresiones poriformes grandes que separan partes más elevadas de aproximadamente el mismo diämetro).

Placa 1' de 1a forma usual en el "grupo tamarensis"; bordes antero-izquierdo y póstero-derecho cortos. Borde antero-derecho de suave concavidad; hacia el comienzo de su cuarto posterior presenta un ojal redondeado y marginal o bien relativamente alejado del borde pero unido e él por un trazo que indica el cerramiento de una muesca original; margen del poro algo espesado. El extremo posterior de la placa varía: en unos casos forma una concavidad de cierta amplitud que se une a 1 a convexidad de 1 a $S . a$. y en otros se prolonga en un pequeño segmento bastante estrecho. La misma variación se nota en el extremo anterior pues puede conectarse con $P_{0}$ una breve truncadura oblicua o puede terminar en punta y sin conexión directa con Po, de 1a que queda separada por una corta unión de $2^{\prime}$ y $4^{\prime}$.

Las restantes apicales son grandes. $2^{\prime}$ y $3^{\prime}$ hexagonales (1a $3^{\prime}$ es mäs ancha y algo más corta). 4' pentagonal muy asimétrica y algo alargada.

La ültima precingular (6'') es relativamente angosta.

En la hipoteca $1 \mathrm{a} 1^{\prime \prime}$ ' es poco o na- da visible en la teca íntegra por su gran oblicuidad aunque es bastante ancha, grande, oval irregular con polo menor anterior, bastante puntiagudo y polo mayor o posterior más o menos aplanado. 6''' casi triangular y más bien estrecha. Su ángulo antero-interno tiene un refuerzo desdoblado. Su borde interno sostiene una aleta muy angosta.

La placa 1'''' es más bien estrecha, muy afinada adelante. Su borde interno, de convexidad suave, sostiene una aleta angosta. 2''' pentagonal (dos bordes ventrales) aproximadamente tan ancha como larga.

La S.a. tiene una forma peculiar y es bastante curvada sobre su eje longitudinal, 1o que dificulta la apreciación de su forma real. Consta de una parte (la miţad o poco menos) anterior algo irregularmente triangular convexa con vértice anterior más o menos redondeado. La mitad posterior es una especie de gancho o de hoz de rama derecha de1gada y puntiaguda, poco más larga que la izquierda (mango de la hoz) que es ancha y algo irregular. Entre ambas ramas queda delimitada una escotadura profunda, bastante estrecha. Las partes anterior y posterior de esta placa estän separadas por un espesamiento transversal muy fuerte, bien visible en la teca integra y que parece prolongar el borde anterior izquierdo del cingulum. Añado que la mitad anterior de la placa es más ventral que la posterior.

La S.p. es algo más larga que ancha, algo trapécicay tiene en la mitad ante-rior una parte hundida triangular a vértice agudo posterior.

Sobre 1a S.p. descansan 1a S.d.p. y 1a S.s.p. La primera es más grande y comienza un poquito más atrás; tiene un borde izquierdo con un refuerzo que termina formando una pequeña protuberancia anterior. LaS.s.p. es pentagonal con un borde posterior, dos laterales y dos anteriores oblicuos, más cortos, sobre todo e1 derecho.

Por arriba de la S.d.p. está la S.d.a., especie de placa $T$ que parece una projecciōn del extremo derecho del cingulum; trapezoidal con borde anterior curvo, ob1icuo y reforzado. La S.S.a. es bastante más pequeña, pentagonal irregular, más alta que ancha, con refuerzos.

Entre las dos sulcales 1aterales anteriores hay una pequeña plaquita bacilar que es la S.m.d. Un poco nás adelante y a la izquierda se encuentra otra placa mucho más grande, casi del tamaño de 1 a 
Lämina 3 - Gonyaulax kutnerae. Fig. 1, vista ventral; Fig. 2, dos tipos de uniön de Po y l'; Fig. 3, placas 2', 3', 4' y Po; Fig. 4, vista antapical; Fig. 5, vista apical; Fig. 6, dos placas l'''; Fig. 7, dos S.a.; Fig. 8, placa 6'''; Fig. 9, sulcales; Fig. 10, una S.P.; Fig. 11, placa 1'i' $(=P$, auct. $)$.

Figura 1 a aproximadamente $\times 700$, Figs 4 y 5 , a aproximadamente $\times 500$; las demás a aumentos mayores no calculados.
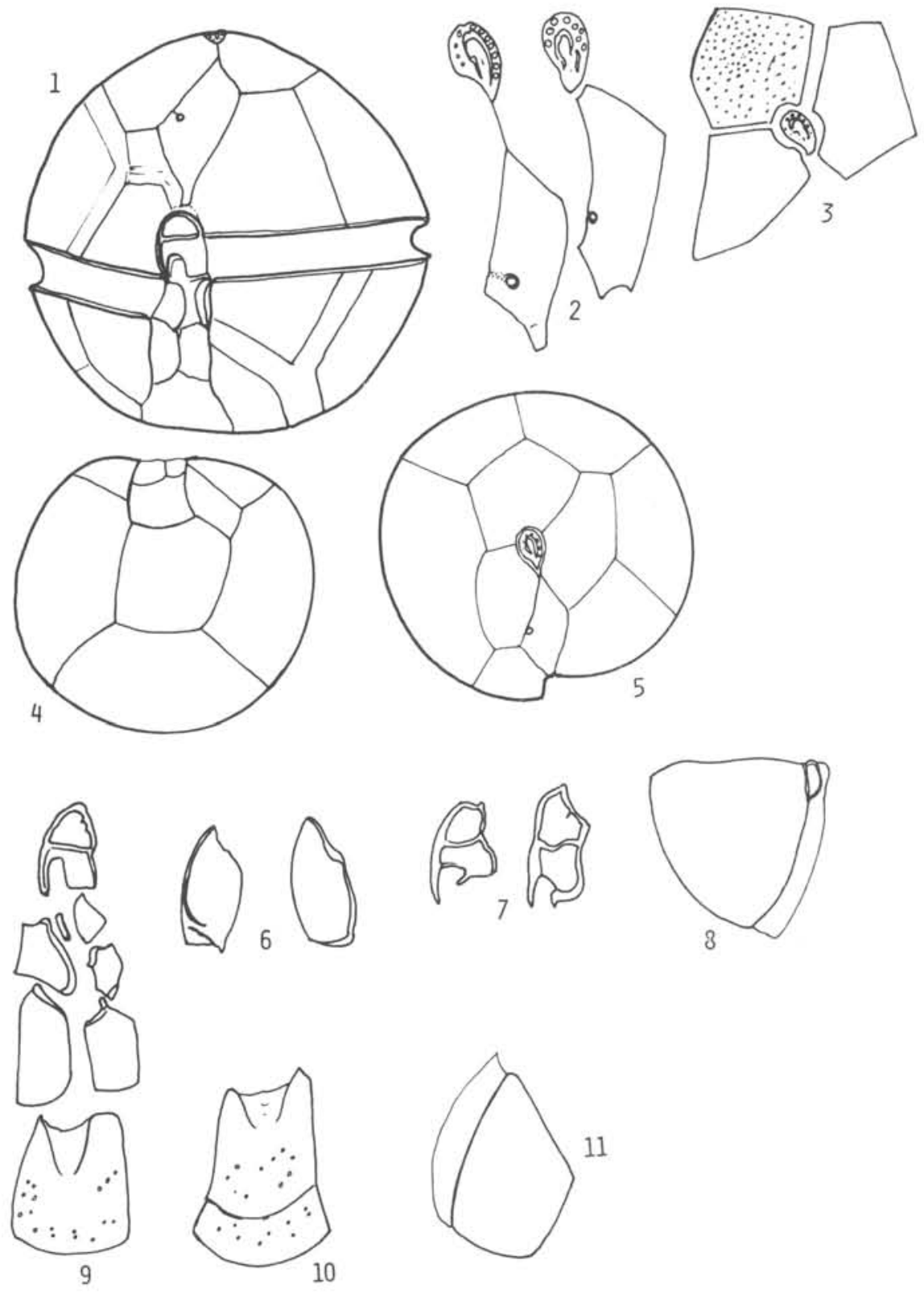

S.s.a., trapécica o triangular con su borde mayor convexo: es la S.m.a.

La teca es débil, mucho más de 10 que suelen ser las de las otras especies del tipo tamarensis. Su escultura está formada por poros bastante densos que a veces recuerdan un poco la escultura de Pyrophacus.

Dimensiones: L 62-65; Trd, 51-57 (ancho mayor unos $6 \mu$ más).

Discusiōn

Por su tamaño, teca débil y forma se di- ferencia netamente de las otras especies del grupo. La muesca de la placa 1', común a la mayoría de las especies de este complejo, aquí se convierte en un ojal cerrado que hasta puede alejarse del borde derecho de la placa. La única especie a la que podrían atribuirse estos ej emplares sería G. brevisulcatum Dangeard $(1927 b)$, pero la descripción de Dangeard es tan incompleta y sus dibujos tan imperfectos que parece arriesgado atribuirle cualquier teca de Gonyaulax que se encuentre; el dibujo muestra la cara 
ventral vista por transparencia desde el dorsso y prácticamente no hay tabulación señalada: la que sería 1 ' parece algo muy diferente de todas 1 as especies del grupo. E1 cingulum tiene un desplazamiento relativamente grande $(2-2,5)$; no hay ninguna indicación de S.a., generalmente muy señalada en el grupo tamarensis $y$, si como supongo, la cara ventral está vista por el lado dorsal (de 1o contrario tendría cingulum ascendente lo que no ocurre en ningún Goniaulax) 1a 1' 'es anorma1mente grande. E1 sulcus sería cortísimo pero probablemente no computó la S.p.

En realidad no hay siquiera una mediocre seguridad de que se trate de un Gonyaulax. Agrego que el hundimiento ventral que muestra la figura del corte óptico publicada por Dangeard no se encuentra en ninguna especie de1 tipo tamarensis.

A pesar de 1o dicho Taylor (1976) corrió el riesgo de atribuir unas tecas de1 0céano Indico, estudiadas por é1, a $G$. brevisulcatum, suponiendo además que G. paulseni Gaarder es la misma especie. Si la identificación y la sinonimia expuesta por Taylor es correcta 10 que primero queda en claro es que brevisulcatum y kutnerae son muy diferentes: las placas $1^{\prime}$ son muy distintas y, los ejemplares de Taylor tienen $6^{\prime \prime}$ y $6^{\prime \prime}$ ' anchas, al parecer carecen de una placa S.a. con la característica forma de garfio y, más importante que todo, su fórmula tabular epitecal no corresponde a ningún Gonyaulax; 1a existencia de tres intercalares y de 7 precingulares hacen que probablemente deban ser transferidos a Peridiniella. Añado que tanto Dangeard como Taylor muestran tecas con cingulum no excavado.

En definitiva esta nueva especie resulta muy claramente distinta de todas 1 as conocidas.

Un hecho importante a destacar es que en $G$. kutnerae la placa $1^{\prime}$ puede estar en contacto o no con Po. En el caso de las tecas en que Po está separada de $1^{\prime}$ este carácter las acerca a G. monilata y G. balechii y, a1 hallarse los dos tipos en la misma especie confirma que el contacto o no contacto entre $P_{0}$ y $1^{\prime}$ es un caräcter secundario que no puede tenervalor genérico, como 10 sugirió primero Howe11 (1953) y luego yo mismo (1964) al hacer notar que en $G$. fratercula $1^{\prime}$ puede estar separada de $P_{0}$ por una distancia muy pequeña, o conectada por una prolongación filiforme. Insistí en que no me parecía que ese contacto tuviese valor genérico en 1977. E1 hallazgo de estos ejemplares demues- tra, al parecer definitivamente, esa tesis. Por lo tanto no podemos aceptar la sugerencia de Taylor de que las especies en que dichas placas no se conectan pertenecen a otro género, y menos a Pyrodinium, que si bien es bastante próximo a Gonyaulax tiene ciertos caracteres propios.

En la muestra 97 del último crucero.

Dedico esta especie a la Dra. Miryam B. Kutner, fitoplanctóloga del Instituto Oceanogräfico da Universidade de São Paulo, quiem me permitió revisar este interesante material.

\section{Resumen}

E1 autor describe dos nuevas especies: Protoperidinium steidingerae y Gonyaulax kutnerae. La tercera especie que se incluye en el estudio, Protoperidinium norpacense Balech, es poco conocida y no se había señalado en el Atlántico. Las tres fueron obtenidas en el litoral sudbrasileño.

La descripción actual de $P$. norpacense completa la primera y muestra ciertas diferencias con la correspondiente a material del Pacífico. Se discuten sus relaciones con $P$. heteracanthum Dangeard.

$P$. steidingerae fue ya descrita por Steidinger \& Williams (1970) como $P$. oblongum. Se ubica próxima a $P$. venustum pero tiene varios caracteres muy peculiares: apical puntiagudo; placa $1^{\prime}$ más corta que la epiteca; placas $1^{\prime}$ y $3^{\prime}$ que no alcanzan al ápice donde aparentemente faltan $P_{0}$ y $X y$, por último, que hay un amplio contacto ventral entre $2^{\prime}$ y $4^{\prime}$.

Gonyaulax kutnerae pertenece al "grupo tamarensis"; su tamaño, forma y teca dëbil la diferencian de las otras especies del grupo. La única que tiene cierto parecido con ella es G. brevisulcatum Dangeard que es casi un nomen nudum. Recientemente Taylor describió como G. brevisulcatum unas tecas que difieren mucho de $G$. kutnerae y que parece ser una Peridiniella, no un Gonyaulax.

\section{Bibliografia}

BALECH, E. 1962. Tintinnoinea y Dinoflagellata del Pacífico según material de las expediciones NORPAC y DOWNWIND de1 Instituto Scripps de Oceanografía. Revta Mus. argent. Cienc. nat. Bernardino Rivadavia Inst. nac. Invest. Cienc. nat., Cienc. Zoo1., 5(1):1-253. 
BALECH, E. 1964. E1 plancton de Mar del Plata durante el período 19611962. Boln Inst. Biol. mar., Mar del Plata, (4):1-50, 5 1ám.

1974. E1 género Protoperidinium Bergh, 1881 ("Peridinium Ehr. 1831, partim). Revta Mus. argent. Cienc. nat. Bernardino Rivadavia Inst. nac. Invest. Cienc. nat., Hidrobiol., $4(1): 1-79$.

1977. Cuatro especies de Gonyaulax sensu lato y consideraciones sobre el género (Dinoflagellata). Revta Mus. argent. Cienc. nat. Bernardino Rivadavia Inst. nac. Invest. Cienc, nat., Hidrobio1., 5(6):115136,3 1ám.

DANGEARD, P. 1972a. Péridiniens nouveaux ou peu connus de 1 a croisiēre du "Sylvana". Bull. Inst. océanogr. Monaco, (491):1-16.

1927b. Phytoplancton de la croisière du "Sylvana". Annls Inst. océanogr. Monaco, n.s., 4(8):285-407.
GAARDER, K. R. 1954. Dinoflagellatae from the "Michael Sars" North Atlantic Deep-Sea Expedition, 1910. Rep. scient. Results Michael Sars N. Atlant. deep Sea Exped., 2(3):1-63, 5 tab.

HOWELL, J. F. 1953. Gonyaulax monilata sp. nov., the causative dinoflagellate of red tide on the east coast of F1orida in August-Sept. 1951. Trans . Am. microsc. Soc., 82(2):153-156.

MATZENAUER, L. 1933. Die Dinoflagellaten des indischen Ozeans. Bot. Arch., $35(4): 437-510$.

STEIDINGER, K. A. 1971. Gonyaulax balechii, sp. nov., with a discussion on the genera Gonyaulax and Heteraulacus. Phycologia, 10(2/3):183-187.

\section{\& WILLIAMS, J. 1970.}

Dinoflagellates. Mem. Hourglass Cruises, Mar. Res. Lab., St. Petersburg, Florida, 2:1-251.

TAYLOR, F. J. R. 1976. Dinoflagellates from the International Indian Ocean Expedition. Biblthca bot., (132):1234, 46 1ām.

(Recibido em 23/noviembre/1978) 